\title{
EVALUATION OF URINARY NUCLEAR MATRIX PROTEIN AS A NEW MARKER FOR URINARY BLADDER CANCER
}

\author{
Takanori Suzuki, Kouichi Suzuki, Hiroshi Okazaki, \\ Yoshihiro Ono, Masashi Ozawa \\ Department of Urology, Gunma Cancer Center
}

\begin{abstract}
The purpose of this study was to evaluate urinary nuclear matrix protein 22 (NMP22) as a marker for urinary bladder cancer. Four groups of subjects participated in this study : 18 with a history of bladder cancer without tumors, 12 with urinary bladder tumors, 9 with prostate cancer, and 2 with renal cell cancer. All patients provided a urine sample for urinary NMP22. All bladder cancer patients provided urine cytology before the cystoscopic examination. The mean urinary NMP22 value was 4.6U/ $\mathrm{ml}$ in bladder cancer patients without tumors, $190.8 \mathrm{U} / \mathrm{ml}$ in bladder cancer patients with tumors, and $5.8 \mathrm{U} / \mathrm{ml}$ in patients with other diseases. The NMP22 value in bladder cancer patients with tumors was significantly higher than that in the other groups. The sensitivity and specificity of urinary NMP22 in urinary bladder cancer was $75 \%$ and $100 \%$, respectively, and the sensitivity of NMP22 was higher than that of urine cytology. This study indicates that urinary NMP22 is a sensitive, non-invasive marker for detecting urinary bladder cancer.
\end{abstract}

Key words : Bladder cancer, Nuclear matrix, Tumor marker

(Kitakanto Med.J. 50 (3) : 237 239, 2000)

\section{INTRODUCTION}

To detect urinary bladder cancer among patients with microscopic hematuria, many subjects would be required to undergo an interventional investigation, such as cystoscopy. However, cystoscopy is relatively invasive. Although the incidence of urothelial cancer among patients with microscopic hematuria is relatively low, it is important to eliminate urinary bladder cancer as a cause of the hematuria. Voided urine cytology is the only non-invasive method that may be practically used for screening urinary bladder cancer patients, but its sensitivity is not sufficiently high ${ }^{1}$. Therefore, development of a more sensitive and simple examination method is needed for screening patients with microscopic hematuria.

It has recently been reported that the nuclear matrix protein 22 (NMP22) was observed in the urine of patients with urothelial cancer ${ }^{2,3)}$. NMP22 is a nuclear matrix protein that may be a useful marker for screen- ing patients with urinary bladder cancer. This study demonstrates that urinary NMP22 is a useful diagnostic marker for urinary bladder cancer.

\section{MATERIALS AND METHODS}

Forty-one patients entered this study and were divided into four groups. Group 1 consisted of 18 patients with a history of bladder cancer who were followed-up without recurrence. Group 2 was 5 patients newly diagnosed with urinary bladder cancer and 7 patients with a recurrence. Group 3 was 9 patients with prostate cancer that were well treated by hormonal therapy. Group 4 was 2 patients with renal cell cancer. All bladder cancer patients were diagnosed by cystoscopy. When bladder tumors were diagnosed by the cystoscopic examination, tumors were transurethrally resected and specimens were pathologically examined. The tumor grade and stage were pathologically and radiographically diagnosed.

Midstream urine in all patients was collected for 
urinary NMP22 and before the cystoscopic examination in the bladder cancer patients. Five $\mathrm{ml}$ of urine was immediately stored in a urine collection Kit containing urine stabilizers at less than $-20^{\circ} \mathrm{C}$. A Konica-Matritech UNMP22 test kit was used to measure the urinary NMP22, which was provided by BML (Biomedical Laboratories, Tokyo). The cut-off value for NMP22 was set at $12 \mathrm{U} / \mathrm{ml}^{4}$. Voided urine cytology was examined in all bladder cancer patients. According to Papanicolaou's classification, classes I, II and III were regarded as negative, and classes IV and V were regarded as positive according to the urinary cytodiagnosis ${ }^{4}$.

Sensitivity and specificity of urinary NMP22 were tested, and the statistical analysis was by the MannWhitney U-test.

\section{RESULTS}

Thirty patients with urinary bladder cancer, consisting of 26 males and 4 females, ranged between 50 and 88 years-old (mean ages, 71.1 years old), and nine with prostate cancer ranged between 47 and 86 years-old (mean ages, 71.1 years old). Of the 30 bladder cancer patients, 12 had urinary bladder tumors and 18 , who had previously received transurethral resection and followed-up, had no tumors. The prostate cancer was treated if the PSA (prostate-specific antigen) value was less than $0.01 \mathrm{ng} / \mathrm{ml}$.

\section{Urinary NMP22 value and its positive rate}

Figure 1 shows the relationship between urinary NMP22 values and diseases. Median values of urinary NMP22 in groups 1, 2, 3, and 4 were 4.6, 190.8, 5.7 and $5.8 \mathrm{U} / \mathrm{ml}$, respectively. Urinary NMP22 values in bladder cancer patients with bladder tumors were significantly higher than those in the other groups $(\mathrm{p}<$

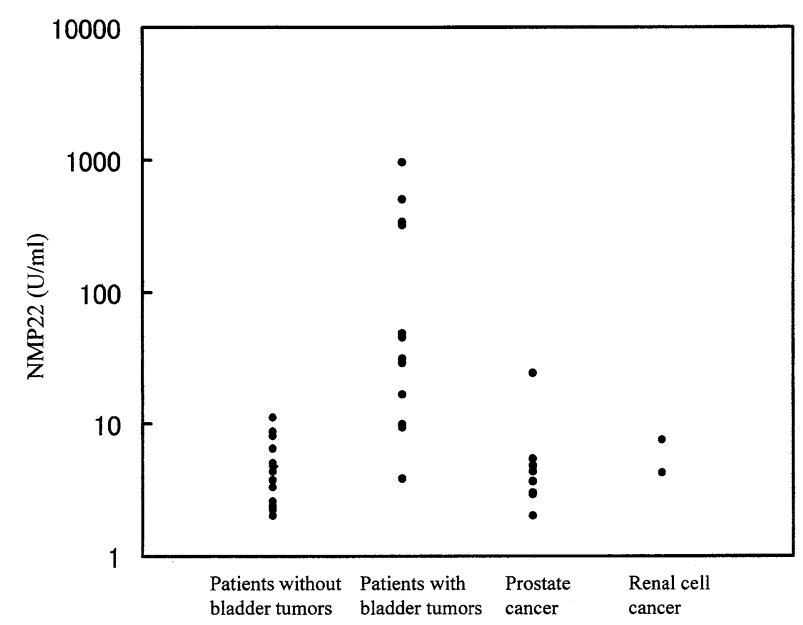

Fig. 1 Urinary NMP22 levels in subjects without bladder tumors and those with bladder tumors, prostate cancer, and renal cell cancer.
0.05 ). The positive rate for urinary NMP22 in bladder cancer patients without tumors, with tumors, and with prostate and renal cell cancer was $0 \%(0 / 18), 75 \%(7 /$ $12)$, and $9.1 \%(1 / 11)$, respectively. The positive rate for bladder cancer patients with bladder tumors was significantly higher than that for patients without bladder tumors with other diseases.

\section{Relationship with urinary NMP 22 and voided urine cytology}

The relationship between urinary NMP22 and voided urine cytology was examined in all bladder cancer patients (Table 1). Four patients with tumors were positive for both urinary NMP22 and urine cytology, but five patients with tumors were positive for only urinary NMP22. Two patients with tumors showed no positive diagnosis for both urinary NMP22 or urine cytology. Sensitivity and specificity of urinary NMP22 and urine cytology were $75 \%$ and $100 \%$, and $33.3 \%$ and $100 \%$, respectively. The positive rate among tumor size, tumor number, stage and grade was examined. The positive rate of urinary NMP22 was higher among all factors than that of urine cytology.

Table 1 Relationship of urinary NMP22 and urine cytology in urinary bladder cancer patients

\begin{tabular}{lccc}
\hline & \multicolumn{3}{c}{ Urine cytology } \\
\cline { 2 - 4 } NMP22 $(\mathrm{n})$ & Positive & Negative & Total \\
\hline Positive & 4 & 5 & 9 \\
Negative & 0 & 21 & 21 \\
Total $(\mathrm{n})$ & 4 & 26 & 30 \\
\hline
\end{tabular}

NMP22, nuclear matrix protein $22 ; n$, number of patients.

Table 2 Relationship of positive rate between NMP22 and urine cytology among patients with urinary bladder cancer

\begin{tabular}{llcc}
\hline & & \multicolumn{2}{c}{ Positive (\%) } \\
\cline { 3 - 4 } & $\mathrm{n}$ & & $\mathrm{UC}$ \\
\hline Tumor size & & 57.1 & 28.6 \\
$\quad<1 \mathrm{~cm}$ & 7 & 66.7 & 33.3 \\
$1-3 \mathrm{~cm}$ & 3 & 100 & 50 \\
$3 \mathrm{~cm}<$ & 2 & & \\
Tumor number & & 75 & 25 \\
1 & 8 & 75 & 50 \\
$2-4$ & 4 & & \\
Tumor stage & & 66.7 & 33.3 \\
Tis-a & 3 & 66.7 & 16.7 \\
T1 & 6 & 100 & 66.7 \\
T2-4 & 3 & & \\
Tumor grade & & 75 & 0 \\
1 & 4 & 71.4 & 42.9 \\
2 & 7 & 100 & 100 \\
3 & 1 & &
\end{tabular}

NMP22, nuclear matrix protein 22; UC, urine cytology; $n$, number of patients. 
Urinary NMP 22 especially showed a higher positive rate for sizes smaller than $1 \mathrm{~cm}$, one tumor and grade 1 tumors.

\section{DISCUSSION}

The prognosis of superficial urinary bladder cancer was better than that of the invasive disease. Although 70 to $80 \%$ of newly diagnosed tumors will have superficial diseases, stage and/or grade progression occurs in a significant number of patients ${ }^{5}$. If tumors are superficial, they can continue to be treated transurethrally. The standard of care for the early detection of recurrent bladder cancer in our hospital is cytology and cystoscopy at 3-month intervals for 3 years after the initial diagnosis or the last tumor recurrence. The sensitivity of urine cytology is low and insufficient for diagnosing urinary bladder cancer. Moreover, cystoscopy is invasive. A new tumor marker for urinary bladder cancer that would reliably predict the presence of transitional cell carcinoma should be developed.

Nuclear matrix proteins are part of the internal structural framework of the nucleus, which may have important roles in deoxyribonucleic acid replication, transcription and processing of ribonucleic acid, and the regulation of gene expression ${ }^{2}$. Nuclear matrix proteins, released from cells after apoptosis, are expressed at 10 to 25 -fold higher levels in neoplastic cells than in normal cells ${ }^{6,7)}$. NMP22 is a protein recognized by the two monoclonal antibodies Mab302-22 and Mab302-18. In addition, these two antibodies recognize the NuMA (nuclear mitotic apparatus) in the nucleus ${ }^{4}$. The NMP22 test kit is specific for the nuclear matrix protein/nuclear mitotic apparatus protein. The assay was designed to measure the nuclear mitotic apparatus protein in the soluble fraction of voided urine ${ }^{2)}$. Sensitivity and specificity of urinary NMP 22 are reported to be 53 to $100 \%$, and 60 to 90 . $1 \%$ for urinary bladder cancer, respectively $y^{4,5,8,99}$. Sensitivity and specificity of urine cytology was 33 to $44 \%$, and 95 to $100 \%$, respectively ${ }^{4,8,9)}$. In this study, sensitivity of urinary NMP 22 was $75 \%$, a higher value than that of urine cytology. The sensitivity of urinary NMP22 in patients with stage Ta-1 tumors, grade 1 tumors, tumor sizes less than $1 \mathrm{~cm}$, and those with only one tumor, was 39.6 to $100 \%, 30.8$ to $33.3 \%, 42.3 \%$, and $47 \%$, respectively ${ }^{4,5}$. This study showed that the positive rate of urinary NMP22 in the above tumor categories was higher than that of urine cytology. Urinary NMP22 was more sensitive for low risk tumors, which is considered to be useful for screening urinary bladder cancer.

The median value of urinary NMP 22 is reported to be $4.4 \mathrm{U} / \mathrm{ml}$ in prostate cancer and $6.2 \mathrm{U} / \mathrm{ml}$ in renal cell cancer $^{4)}$. In normal healthy volunteers and in subjects with benign conditions, the median NMP22 levels were 2.9 and $3.3 \mathrm{U} / \mathrm{mL}$, respectively ${ }^{2}$. Our results were similar to those. Moreover, urinary NMP 22 is thought to be more specific for urinary bladder cancer. Urinary NMP22 in the midnight-to-noon single void and the pooled 3-void sample was reported to be similar for predicting a recurrence postoperatively in patients with a urinary tract transitional cell carcinoma $^{3)}$. A single void collection provides a simpler and more cost-effective sampling method. In addition, the urinary NMP22 assay may be a useful adjunct for the urologist who treats patients with urinary bladder cancer.

\section{REFERENCES}

1) Miyanaga $N$, Akaza $H$, Tsukamoto $T$, et al. Urinary nuclear matrix protein 22 as a new marker for the screening of urothelial cancer in patients with microscopic hematuria. Int J Urol 1999; 6 : 173-177.

2) Carpinito GA, Stadler WM, Briggman JV, et al. Urinary nuclear matrix protein as a marker for transitional cell carcinoma of the urinary tract. $\mathrm{J}$ Urol $1996 ; 156: 1280-1285$.

3) Chen YT, Hayden CL, Marchand KJ, et al. Comparison of urine collection methods for evaluating urinary nuclear matrix protein, NMP22, as a tumor marker. J Urol 1997; 158 : 1899-1901.

4) Akaza $\mathrm{H}$, Miyanaga $N$, Tsukamoto $T$, et al. Evaluation of urinary NMP22 (nuclear matrix protein) as a diagnostic marker for urothelial cancer - NMP22 as a urinary marker for surveillance of bladder cancer. Jpn J Cancer Chemother 1997; 24 : 829-836.

5) Stampfer DS, Carpinito GA, RodriguezVillanueva J, et al. Evaluation of NMP22 in the detection of transitional cell carcinoma of the bladder. J Urol 1998; 159: 394-398.

6) Badalament RA. Is the role of cystoscopy in the detection of bladder cancer really declining? J Urol 1998; 159 : 399-400.

7) Hoffman M. The cell's nucleus shapes up. Science $1993 ; 259$ : 1257-1259.

8) Zippe C, Pandrangi L, Agarwal A. NMP22 is a sensitive, cost-effective test in patients at risk for bladder cancer. J Urol 1999; 161 : 62-65.

9) Ramakumar S, Bhuiyan J, Besse JA, et al. Comparison of screening methods in the detection of bladder cancer. J Urol 1999; 161 : 388-394. 